\title{
Verzeichnis der Siglen für wissenschaftliche Zeitschriften, Reihen und Sammelwerke
}

AAkGött
AASF
ABäG
ABayA
ABgesch
ABnG
AdA
AfK
AGB
AGSZ

Ahd. St. Gallen

Ak. Wiss.

DDR. ZS.

ALASH

AmL

APreußA

ASNS

ASSL

ASTH

AT

ATB

Aufriß

AUS

AWMainz

AzF
BAS
BBG
BdPh
B-EBS
BGermNat

B. Gesch. Nhd.

BLM

BLV

BNF

BNL

BSGLN

CILT

Clex.

DaF

DD

DDG

DGF

DLE

ds

DSA
Abhandlungen der Akademie der Wissenschaften in Göttingen. Phil.-Hist. Klasse

Suomalaisen Tiedeakatemian Toimituksia. Annales Academiae Scientiarum Fennicae. Series B Amsterdamer Beiträge zur älteren Germanistik

Abhandlungen der Bayerischen Akademie der Wissenschaften. Phil.-Hist. Klasse

Archiv für Begriffsgeschichte

Amsterdamer Beiträge zur neueren Germanistik

Anzeiger für deutsches Altertum und deutsche Literatur

Archiv für Kulturgeschichte

Archiv für Geschichte des Buchwesens

Abhandlungen, hrsg. von der Gesellschaft für deutsche Sprache in Zürich

Das Althochdeutsche von St. Gallen

Akademie der Wissenschaften der DDR. Zentralinstitut für Sprachwissenschaft

Acta Linguistica Academiae Scientiarum Hungaricae

Arbeiten zur mittleren deutschen Literatur

Abhandlungen der Preußischen Akademie der Wissenschaften

Archiv für das Studium der neueren Sprachen und Literaturen

Archiv für das Studium der neueren Sprachen und Literaturen

Amsterdam Studies in the Theory and History of Linguistic Science

Athenäum Taschenbücher Sprachwissenschaft

Altdeutsche Textbibliothek

Deutsche Philologie im Aufriß. 2. Auflage

Acta Universitatis Stockholmiensis

Akademie der Wissenschaften und der Literatur Mainz. Abhandlungen der Geistes- und sozialwissenschaftlichen Klasse

Arbeiten zur Frühmittelalterforschung

Bibliothek der Allgemeinen Sprachwissenschaft

Budapester Beiträge zur Germanistik

Beiträge zur deutschen Philologie

Bochum-Essener Beiträge zur Sprachwandelforschung

Bibliothek des Germanischen Nationalmuseums Nürnberg zur deutschen Kunst- und Kulturgeschichte

Bausteine zur Geschichte des Neuhochdeutschen

Bibliographien zur deutschen Literatur des Mittelalters

Bibliothek des Litterarischen Vereins in Stuttgart

Beiträge zur Namenforschung

Bibliothek der gesammten deutschen National-Literatur von der ältesten bis auf die neuere Zeit

Bouwstoffen en Studien voor de Geschiedenis en de Lexikografie van het Nederlands

Current Issues in Linguistic Theory

Cahiers de Lexicologie. Revue internationale de lexicologie générale et appliquée

Deutsch als Fremdsprache. Zeitschrift zur Theorie und Praxis des Deutschunterrichts für Ausländer

Diskussion Deutsch. Zeitschrift für Deutschlehrer aller Schulformen in Ausbildung und Praxis

Deutsche Dialektgeographie

Dokumentation Germanistischer Forschung

Deutsche Literatur in Entwicklungsreihen

deutsche sprache. Zeitschrift für Theorie, Analyse und Dokumentation

Deutscher Sprachatlas. Marburg 1927-1956 
DSF

Dt. Ak. Wiss.

Berlin, IDSL

DTM

DU

DVLG

DWA

DWB

DWEB

EB

Euph.

FAT

FAZ

FF

FIdS

FMA

FoL

FSt

GA

GASK

GdK

GdL

GeLe

GG

GGA

GL

\section{GRM}

Grundr.

GS

GSR

HBV

HCTD

Hesperia

HGF

$\mathrm{HJb}$

HSK

HSS

HW

$\mathrm{HZ}$

IBAL

IBK

IBS

IdB

IdL

IF

IJB

IJL

IVaS

$\mathrm{JgF}$

JIdS

JIG

JL SMa.

JL SMi.

JL SPr.
Deutsch-slawische Forschungen zur Namenkunde und Siedlungsgeschichte

Deutsche Akademie der Wissenschaften zu Berlin. Veröffentlichungen des Instituts für

Deutsche Sprache und Literatur

Deutsche Texte des Mittelalters

Der Deutschunterricht. Beiträge zu seiner Praxis und wissenschaftlichen Grundlegung

Deutsche Vierteljahresschrift für Literaturwissenschaft und Geistesgeschichte

Deutscher Wortatlas

Deutsches Wörterbuch von Jacob Grimm und Wilhelm Grimm

Deutsche Wortforschung in europäischen Bezügen

Eichstätter Beiträge. Schriftenreihe der Katholischen Universität Eichstätt

Euphorion. Zeitschrift für Literaturgeschichte

Fischer Athenäum Taschenbücher

Frankfurter Allgemeine Zeitung

Forum für Fachsprachenforschung

Forschungsberichte des Instituts für deutsche Sprache

Forschungen und Materialien zur deutschen Aufklärung

Folia Linguistica. Acta Societatis Linguisticae Europeae

Frühmittelalterliche Studien

Germanistische Abhandlungen

Germanistische Arbeiten zu Sprache und Kulturgeschichte

Grundlagen der Kommunikation

Geschichte der deutschen Literatur von den Anfängen bis zum Beginn der Neuzeit

Germanistische Lehrbuchsammlung

Grundlagen der Germanistik

Göttingische Gelehrte Anzeigen

Germanistische Linguistik. Berichte aus dem Forschungsinstitut für deutsche Sprache, Deutscher

Sprachatlas

Germanisch-Romanische Monatsschrift

Grundriß der Germanischen Philologie

Germanische Studien

Göttinger Studien zur Rechtsgeschichte

Hessische Blätter für Volkskunde

Handelingen van de Koninklijke Commissie voor Toponymie en Dialectologie

Hesperia. Schriften zur germanischen Philologie

Hermaea. Germanistische Forschungen

Historisches Jahrbuch

Handbücher zur Sprach- und Kommunikationswissenschaft

Historischer Südwestdeutscher Sprachatlas

Historische Wortforschung. Untersuchungen zur Sprach- und Kulturgeschichte des Deutschen in seinen europäischen Bezügen

Historische Zeitschrift

Internationale Bibliothek für Allgemeine Sprachwissenschaft

Innsbrucker Beiträge zur Kulturwissenschaft

Innsbrucker Beiträge zur Sprachwissenschaft

Indices zur deutschen Barockliteratur

Indices zur deutschen Literatur

Indogermanische Forschungen. Zeitschrift für Indogermanistik und allgemeine Sprachwissenschaft

Indogermanisches Jahrbuch

International Journal of Lexicography

Indices verborum zum altdeutschen Schrifttum

Jenaer germanistische Forschungen

Jahrbuch des Instituts für deutsche Sprache

Jahrbuch für Internationale Germanistik

Janua Linguarum, Series Maior

Janua Linguarum, Series Minor

Janua Linguarum, Series Practica 
KBGL

KdPM

$\mathrm{KgSt}$

KSL

KTVÜ

KVndSpr.

KZSS

LA

LB

LBij

LGL

LiLi

LR

LRL

LSM

LStA

LuD

MBG

MBVM

MdF.

MdSt.

MDGV

Med. Aev.

MGB

$\mathrm{MGH}$

MIÖG

ML

MLK

MLQ

MLR

MM-S

MSH

MSpr.

MTU

Mutterspr.

$\mathrm{MzS}$

NdD

$\mathrm{NdJb}$.

$\mathrm{NdM}$

NdSt.

NdW

Nk.

NLS

NphM

NS

Onoma

PBB (H)

PBB (T)

PGRK

Poetica

PSQ

QFSK

rde

RdL
Kopenhagener Beiträge zur Germanistischen Linguistik

Kleinere deutsche Prosadenkmäler des Mittelalters

Kölner germanistische Studien

Konzepte der Sprach- und Literaturwissenschaft

Kleine Texte für Vorlesungen und Übungen

Korrespondenzblatt des Vereins für niederdeutsche Sprachforschung

Kölner Zeitschrift für Soziologie und Sozialpsychologie

Linguistische Arbeiten

Linguistische Berichte. Forschung, Information, Diskussion

Leuvense Bijdragen, Tjdschrift voor Germaanse Filologie

Lexikon der germanistischen Linguistik

Zeitschrift für Literaturwissenschaft und Linguistik

Linguistische Reihe

Lexikon der Romanistischen Linguistik

Lexicographica, Series Maior

Linguistische Studien A. Arbeitsberichte

Linguistik und Didaktik

Marburger Beiträge zur Germanistik

Materialien zur Bibelgeschichte und religiösen Volkskunde des Mittelalters

Mitteldeutsche Forschungen

Mitteldeutsche Studien

Mitteilungen des Deutschen Germanistenverbandes

Medium Aevum. Philologische Studien

Münchner Germanistische Beiträge

Monumenta Germaniae Historica

Mitteilungen des Instituts für österreichische Geschichtsforschung

Modern Languages. Journal of the Modern Language Association

Monographien zur Linguistik und Kommunikationswissenschaft

Modern Language Quarterly

Modern Language Review

Münstersche Mittelalter-Schriften

Mémoires de la Société Néo-Philologique de Helsingfors

Moderna Språk

Münchener Texte und Untersuchungen zur deutschen Literatur des Mittelalters

Muttersprache. Zeitschrift zur Pflege und Erforschung der deutschen Sprache

Monographien zur Sprachwissenschaft

Niederdeutsche Denkmäler

Niederdeutsches Jahrbuch. Jahrbuch des Vereins für niederdeutsche Sprachforschung

Niederdeutsche Mitteilungen

Niederdeutsche Studien

Niederdeutsches Wort. Kleine Beiträge zur niederdeutschen Mundart und Namenkunde

Naamkunde

North-Holland Linguistic Series

Neuphilologische Mitteilungen. Bulletin de la Société Néophilologique de Helsinki

Die neueren Sprachen. Zeitschrift für Forschung, Unterricht und Kontaktstudium auf dem Fachgebiet der modernen Fremdsprache

Onoma. Bulletin d'information et de bibliographie des sciences onomastiques

Beiträge zur Geschichte der deutschen Sprache und Literatur (Halle/S.)

Beiträge zur Geschichte der deutschen Sprache und Literatur (Tübingen)

Publikationen der Gesellschaft für rheinische Geschichtskunde

Poetica. Zeitschrift für Sprach- und Literaturwissenschaft

Philologische Studien und Quellen

Quellen und Forschungen zur Sprach- und Kulturgeschichte der germanischen Völker

rowohlts deutsche enzyklopädie

Reallexikon der deutschen Literaturwissenschaft. Neubearbeitung des Reallexikons der deutschen

Literaturgeschichte 
RF

RGL

RheinBH

RL

$\mathrm{RVj}$.

Sächs. Ak. Wiss.

Leipzig

SaGö

SAV

SbAWiss Wien

SbBayA

SbHeidelbA

SbÖstA

SbWiss.GF

SG

SGF

SGGand.

Skandinavistik

SKGG

SLG

SM

SN

SPÖ

Sprachw.

Spr. d. Geg.

SQwestGesch

ST

StAhd.

StFrnhd.

$\mathrm{StGr}$

STSL

STW

STZ

SU Augsb.

$\mathrm{SuG}$

SuGesch

$\mathrm{SuL}$

TCLC

TCLP

Teuth.

THF

TLL

TLP

TLSM

TMN

TNTL

TT

TTG

VIRM

VjWS

VKLBayA

VL

VMA

VRom
Romanische Forschungen

Reihe Germanistische Linguistik

Rheinische Beiträge und Hülfsbücher zur germanischen Philologie und Volkskunde

Reallexikon der deutschen Literaturgeschichte

Rheinische Vierteljahresblätter. Mitteilungen des Instituts für geschichtliche Landeskunde der

Rheinlande an der Universität Bonn

Berichte über die Verhandlungen der Sächsischen Akademie der Wissenschaften zu Leipzig. Phi-

lologisch-historische Klasse

Sammlung Göschen

Sammlung Akademie Verlag

Sitzungsberichte der Akademie der Wissenschaften in Wien. Phil.-hist. Klasse

Sitzungsberichte der Bayerischen Akademie der Wissenschaften. Philosophisch-historische

Klasse

Sitzungsberichte der Heidelberger Akademie der Wissenschaften

Sitzungsberichte der Österreichischen Akademie der Wissenschaften. Philosophisch-Historische

Klasse

Sitzungsberichte der Wissenschaftlichen Gesellschaft an der Universität Frankfurt

Studium Generale

Stockholmer Germanistische Forschungen

Studia Germanica Gandensia

Skandinavistik. Zeitschrift für Sprache, Literatur und Kultur der nordischen Länder

Schriften der Königsberger Gelehrten Gesellschaft. Geisteswissenschaftliche Klasse

Studia Linguistica Germanica

Sammlung Metzler

Studia Neophilologica. A Journal of Germanic and Romanic Philology

Sprache Politik Öffentlichkeit

Sprachwissenschaft

Sprache der Gegenwart. Schriften des Instituts für deutsche Sprache

Studien und Quellen zur westfälischen Geschichte

Suhrkamp taschenbuch

Studien zum Althochdeutschen

Studien zum Frühneuhochdeutschen

Studia Grammatica

Studien und Texte zur Sozialgeschichte der Literatur

Suhrkamp Taschenbuch Wissenschaft

Sprache im technischen Zeitalter

Schriften der philosophischen Fachbereiche der Universität Augsburg

Sprache und Gesellschaft

Sprache und Geschichte

Sprache und Literatur in Wissenschaft und Unterricht

Travaux du Cercle Linguistique de Copenhague

Travaux du Cercle Linguistique de Prague

Teuthonista

Trierer Historische Forschungen

Travaux de Linguistique et de Litterature

Travaux Linguistiques de Prague

Trends in Linguistics. Studies and Monographs

Texte des späten Mittelalters und der frühen Neuzeit

Tijdschrift voor Nederlandse Taal- en Letterkunde

Taal en Tongval

Texte und Textgeschichte. Würzburger Forschungen

Veröffentlichungen des Instituts für Realienkunde des Mittelalters und der frühen Neuzeit

Vierteljahresschrift für Wirtschafts- und Sozialgeschichte

Veröffentlichungen der Kommission für Deutsche Literatur des Mittelalters der Bayerischen Akademie der Wissenschaften

Die deutsche Literatur des Mittelalters. Verfasserlexikon

Verslagen en Mededelingen van de Koninklijke Academie voor Nederlandse Taal- en Letterkunde [früher: van de Koninklijke Vlaamse Academie voor Taal- en Letterkunde]

Vox Romana 
WB

WBdPh

WdF

WNF

WuS

WW

WZUB

WZUG

WZUJ

WZUL

WZUR

ZbayrLa

ZD

$\mathrm{ZdA}$

$\mathrm{ZdB}$

ZDk.

ZDL

ZdMaa.

$\mathrm{ZdPh}$.

$\mathrm{ZdS}$

ZdU

ZdWf.

ZfRG

ZfS

ZfSI.

ZfVk.

ZGL

ZhdMaa

ZMF

ZNf.

ZONf.

ZPSK

$\mathrm{ZrPh}$.

$\mathrm{ZsPh}$.

ZSRG

Zvgl. Sprachf.
Weimarer Beiträge

Würzburger Beiträge zur deutschen Philologie

Wege der Forschung

Wortbildung des Nürnberger Frühneuhochdeutsch

Wörter und Sachen

Wirkendes Wort. Deutsche Sprache in Forschung und Lehre

Wissenschaftliche Zeitschrift der Humboldt-Universität zu Berlin. Gesellschafts- und sprachwissenschaftliche Reihe

Wissenschaftliche Zeitschrift der Ernst-Moritz-Arndt-Universität Greifswald. Gesellschafts- und sprachwissenschaftliche Reihe

Wissenschaftliche Zeitschrift der Friedrich-Schiller-Universität Jena

Wissenschaftliche Zeitschrift der Karl-Marx-Universität Leipzig

Wissenschaftliche Zeitschrift der Universität Rostock

Zeitschrift für bayrische Landesgeschichte

Zielsprache Deutsch. Zeitschrift für Unterrichtsmethodik und angewandte Sprachwissenschaft

Zeitschrift für deutsches Altertum und deutsche Literatur

Zeitschrift für deutsche Bildung

Zeitschrift für Deutschkunde

Zeitschrift für Dialektologie und Linguistik

Zeitschrift für deutsche Mundarten

Zeitschrift für deutsche Philologie

Zeitschrift für deutsche Sprache

Zeitschrift für den deutschen Unterricht

Zeitschrift für deutsche Wortforschung

Zeitschrift für Rechtsgeschichte

Zeitschrift für Sprachwissenschaft

Zeitschrift für Slawistik

Zeitschrift für Volkskunde

Zeitschrift für Germanistische Linguistik

Zeitschrift für hochdeutsche Mundarten

Zeitschrift für Mundartforschung

Zeitschrift für Namenforschung

Zeitschrift für Ortsnamenforschung

Zeitschrift für Phonetik, Sprachwissenschaft und Kommunikationsforschung

Zeitschrift für romanische Philologie

Zeitschrift für slavische Philologie

Zeitschrift der Savigny-Stiftung für Rechtsgeschichte

Zeitschrift für vergleichende Sprachforschung auf dem Gebiet der indogermanischen Sprachen 